\title{
College of American Pathologists
}

National Cancer Institute

\section{Source}

National Cancer Institute. College of American Pathologists. NCI Thesaurus. Code C157719.

A member-based physician that serves patients, pathologists, and the public by fostering and advocating best practices in pathology and laboratory medicine. 